\title{
Experimental study of electrical breakdown in MEMS devices with micrometer scale gaps
}

\author{
P. Carazzetti, Ph. Renaud, H. R. Shea
}

Copyright 2008 Society of Photo-Optical Instrumentation Engineers.

This paper was published in Proceedings of SPIE Volume 6884 and is made available as an electronic reprint (preprint) with permission of SPIE. One print or electronic copy may be made for personal use only. Systematic or multiple reproduction, distribution to multiple locations via electronic or other means, duplication of any material in this paper for a fee or for commercial purposes, or modification of the content of the paper are prohibited. 


\title{
Experimental study of electrical breakdown in MEMS devices with micrometer scale gaps
}

\author{
P. Carazzetti ${ }^{\mathrm{a}}$, Ph. Renaud ${ }^{\mathrm{b}}$, H. R. Shea ${ }^{{ }_{\mathrm{a}}}$ \\ ${ }^{a}$ LMTS - EPFL, Ecole Polytechnique Fédérale de Lausanne, Rue Jaquet Droz 1, CH-2002 \\ Neuchâtel, Switzerland \\ ${ }^{\mathrm{b}}$ LMIS4 - EPFL, Ecole Polytechnique Fédérale de Lausanne, CH-1015 Lausanne, Switzerland.
}

\begin{abstract}
We present an experimental study of the DC breakdown voltage of MEMS interdigitated aluminum electrodes with gaps ranging from 10 to $500 \mu \mathrm{m}$. Unlike most research on MEMS electrodes that was done at atmospheric pressure, our work has focused on the effect of gas pressure and gas type on the breakdown voltage. A main goal was to identify geometries that favor the creation of low-voltage discharges. Helium, argon and nitrogen pressure was varied from $10^{2}$ to $8.10^{4} \mathrm{~Pa}(1$ to 800 mbar). The breakdown voltage was plotted as a function of the Paschen reduced variable $P_{r e d}=p \cdot d$. For higher values of pressure, $p$ or gap, $d$ (high $P_{r e d}$ ), classical Paschen scaling was observed. For lower values of $P_{\text {red }}$ however, significant deviations were seen, particularly at low pressures. We attribute these differences not to field emission, but to the scale of the mean free path (which explains the higher than predicted voltages), and principally to the many length scales effectively present in our planar geometry (on-chip and even off-chip, that lead to the superposition of several Paschen curves). Guidelines are proposed for low-pressure operation of MEMS to avoid or to encourage breakdown.
\end{abstract}

Keywords: Paschen curve, breakdown voltage, MEMS, planar interdigitated electrodes, aluminum, helium, argon, nitrogen, mean free path

\section{INTRODUCTION}

\subsection{Paschen curves in MEMS devices}

Most of the research on Paschen curves applied to MEMS devices (i.e. to micron-size gaps) has focused on understanding the safe operating conditions in order to design electrostatic actuators operating at high voltages (of order $200 \mathrm{~V}$ ) with gaps between electrodes of order a micron or even less. The limitation of the Paschen curve at micron-scale gaps have become clear in the past few years [1-6]. The importance of the role of field emission and vapor arc have been demonstrated for gaps smaller than 10 microns, leading to the description of the "modified" Paschen curve [4]. The general conclusion has been that a maximum safe voltage is $300 \mathrm{~V}$ for gaps 4 microns or larger at a pressure of one atmosphere, and that the breakdown voltage decreases rapidly for smaller gaps due to field emission [4].

In 1889, F. Paschen published a paper [7] which laid out what has become known as Paschen's Law. The law expresses the breakdown voltage $V_{b d}$ of a dielectric gas as a function of the reduced variable $P_{\text {red }}=p \cdot d$, where $p$ is the pressure and $d$ is the gap (Figure 1A). His work was developed to understand the breakdown voltage between large metal plates at low pressure with macroscopic gaps.

Later work by J. Townsend [8] led to the understanding that the breakdown is an avalanche effect caused principally by the ionization of gas molecules by electrons accelerated by the electric field. If the electron gains sufficient energy between collisions to ionize gas atoms or gas molecules, then each collision gives rise to two electrons and an ion, allowing an avalanche effect eventually resulting in a spark. This avalanche can only occur when there are sufficient gas molecules between the electrodes, i.e. if the mean free path between collisions $\lambda$ is much smaller than the distance $d$ between electrodes: when $\lambda<<$. If the pressure is too low, or if the gap is too small, the avalanche breakdown (Townsend theory) cannot take place.

*herbert.shea@epfl.ch; phone +41 3272055 84; fax +41 3272057 54; http://lmts.epfl.ch

Reliability, Packaging, Testing, and Characterization of MEMS/MOEMS VII, edited by Allyson L. Hartzell, Rajeshuni Ramesham, Proc. of SPIE Vol. 6884, 688404, (2008) · 0277-786X/08/\$18 · doi: 10.1117/12.763195 
This absence of atoms or molecules is what gives the minimum in the Paschen curve. At large gaps or pressures, a linear relation breakdown voltage and electrode gap is found (reflecting the constant breakdown electric field of the gas), while at very small gaps one has a "vacuum isolation", where there are not enough gas atoms or molecules for the avalanche to occur. Later work led to the understanding that the Paschen curve also depends on secondary electrons emitted from the negative electrode when impacted by the positive ions. These electrons further accelerate the breakdown process. The secondary electron yield, $\gamma$ depends on the cathode material $[9,10]$. The Paschen curve can be obtained by computing the voltage required for the process of electron emission and multiplication to become self-sustaining [11]. One obtains:

$$
V_{b d}=\frac{B \cdot p \cdot d}{\ln (A \cdot p \cdot d)-\ln \left(\ln \left(1+\frac{1}{\gamma}\right)\right)}
$$

where $A$ and $B$ are properties of the gas, and $\gamma$ is a property of the electrode material.

The Paschen curves were developed for macroscopic electrodes at operating pressures from a few Pa to one atmosphere. The generality of the scaling of $V_{b d}$ with $P_{\text {red }}$ led researchers to apply it to MEMS devices operating in a variety of gases at one atmosphere, for which a minimum breakdown voltage of order $380 \mathrm{~V}$ is predicted at a spacing of 8 microns (the exact numbers depend on the gas).

As reported in $[1,2,5,6]$, when the gaps are less than 10 microns for micromachined structures operated at one atmosphere $\left(P_{\text {red }}<1 \mathrm{~Pa} \cdot \mathrm{m}\right)$, important deviations are seen from the Paschen curve. This regime is one where the mean free path is of order the gap, and thus where the Townsend breakdown cannot occur. Other types of breakdown are however possible. As presented for instance in [4] and in [6], field emission can become important at gaps order 5 microns, leading to a "modified" Paschen curve, which agrees with the "standard" Paschen curves at gaps larger than 10 microns $\left(P_{r e d}>1 \mathrm{~Pa} \cdot \mathrm{m}\right)$, exhibits a plateau of constant $V_{b d}$ between 4 and 10 microns, and a linear drop in $V_{b d}$ at lower gaps (dotted line on Figure 1A).
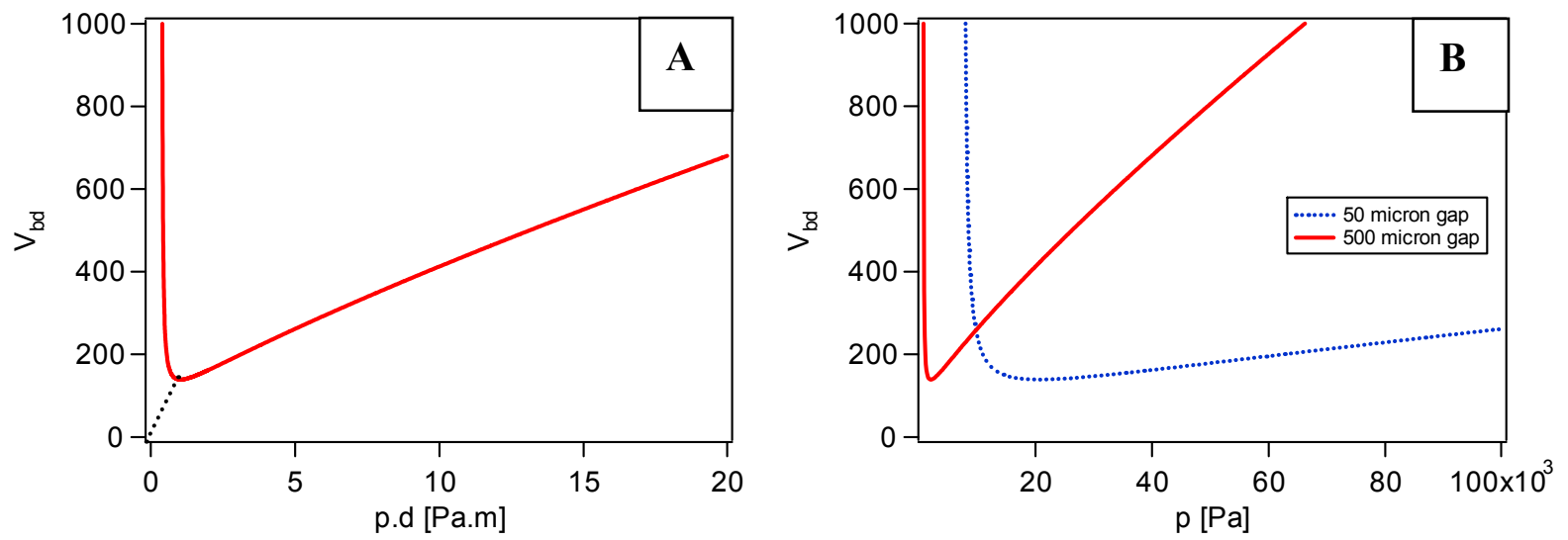

Fig. 1. A) Paschen curve for argon (solid line) and modified Paschen curve (dotted line) showing the drop in $V_{b d}$ at low gaps. B) Paschen curve in argon for gaps of $50 \mu \mathrm{m}$ (dotted line) and $500 \mu \mathrm{m}$ (solid line): the minimum voltage for breakdown is constant but the pressure at which the minimum in $V_{b d}$ occurs is gap dependent.

In addition to the nature of the gas (reflected in the constants $A$ and $B$ of equation 1) and the nature of the electrode (in the form of parameter $\gamma$ ), relevant parameters that must be taken into account are the mean free path of gas atoms species, the surface roughness (which has a strong influence on the field emission), work function of the electrode, and the overall geometry of the electrodes, especially for planar geometries as found in MEMS and integrated circuits, which do not match the conditions of uniform electric field for which the Paschen curves were developed. 


\subsection{Motivation of the research}

With the exception of the work of one group $[10,12,13]$ Paschen curves for micron-scale gaps have been measured at atmospheric pressure $[1,4,14,15]$, typically in air, which is reasonable given that this is the normal operating condition for MEMS devices. Although Baars-Hibbe and coworkers [10] report on measurements down to $10 \mathrm{kPa}$, their main objective was creating atmospheric plasmas.

Our motivation is to develop a chip-scale hermetically sealed plasma light source, consisting of Al electrodes spaced by 10 to 500 microns sealed in a stack of three anodically-bonded glass wafers. We report on the fabrication process and optical spectra obtained from this light source in [16] for different gases and pressures. Applications for such a light source include optical pumping in chip-scale atomic clocks, leak detection, and outgassing characterization.

To ignite the plasma discharge, a sufficient AC or DC potential $(\sim 300 \mathrm{~V})$ must be applied between the electrodes. For lifetime and power consumption reasons, our chip-scale light source is operated as an RF plasma at frequencies near 20 MHz. RF operation minimizes erosion of the cathode (thus increasing lifetime), and can reduce the minimum voltage required for ignition as reported in [13].

The goal of the study reported here was to compare different electrode gap sizes and gap geometries in order to determine the conditions under which a plasma can be ignited with the lowest possible voltage over a wide pressure range (i.e., $10^{2}$ to $8 \cdot 10^{4} \mathrm{~Pa}$, or 1 to $800 \mathrm{mbar}$ ). RF plasmas are more complex to understand than DC plasma, with additional lengths scales that need to be considered, primarily the fact that the electrons are confined by field oscillations, and the space-charge sheaths at the plasma boundary.

We therefore investigated the DC Paschen curve of the structure described in the following section, varying both the gap (from 10 to $500 \mu \mathrm{m}$ ) and the pressure $\left(10^{2}\right.$ to $8 \cdot 10^{4} \mathrm{~Pa}$ ), for $\mathrm{He}, \mathrm{Ar}$ and $\mathrm{N}_{2}$ atmospheres. Our samples thus cover a very large range of $P_{\text {red }}$ : more than 4 order of magnitude from $P_{\text {red }}=10^{-3} \mathrm{~Pa} \cdot \mathrm{m}$ to $40 \mathrm{~Pa} \cdot \mathrm{m}$, covering regimes of small $P_{\text {red }}$ not accessible to devices (regardless of size) operated at atmospheric pressure because of the field emission occurring at small gaps.

Section 2 presents the sample design and fabrication. In section 3 the measurement setup is described, and data is presented on the breakdown voltage as a function of pressure, gas type and gap size. Experimental data is discussed in section 4.

\section{SAMPLE DESIGN AND FABRICATION}

Interdigitated electrodes with 3 pairs of fingers covering two orders of magnitude of gap spacing were designed and fabricated. Figure $2 \mathrm{~A}$ is a CAD plot showing the device layout in micrometers. Each finger is $2820 \mu \mathrm{m}$ long and $240 \mu \mathrm{m}$ wide. Corrugated edges were used to locally enhance the electric field and hence to lower the voltage required for breakdown. The distance separating the tip of opposite protruding patterns, shown by the scale bars in Figure 2B, is referred to, in following text, as the electrode gap spacing, $d$. Varying only the parameter $d, 8$ different electrode versions were designed, namely, $d=10,20,40,100,150,200,300$ and $500 \mu \mathrm{m}$.
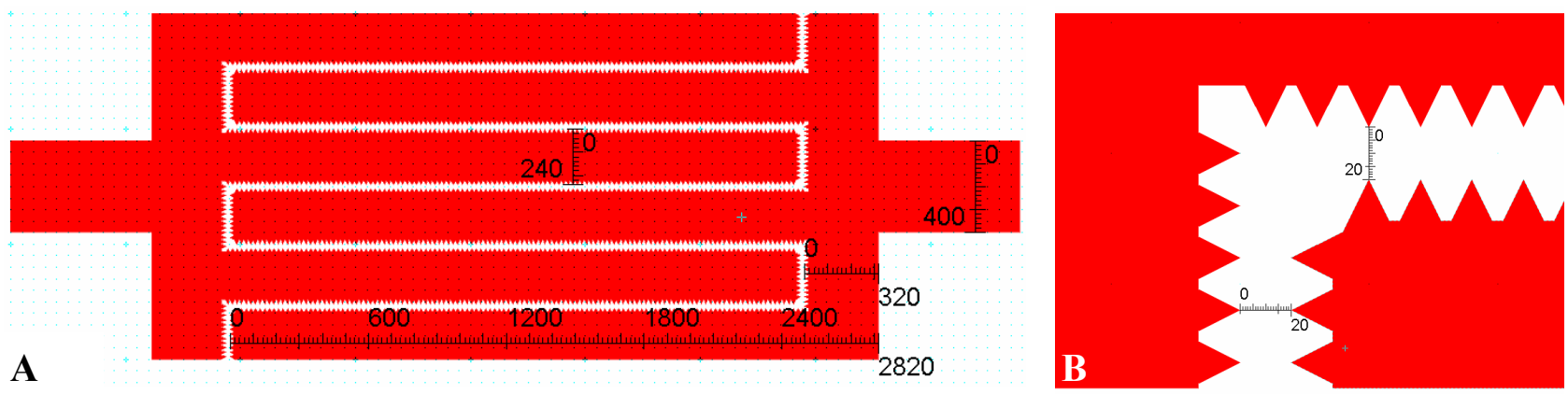

Fig. 2. A) CAD layout showing the overall footprint of a device. B) Close-up showing the 20- $\mu$ m gap separation between opposite protruding patterns. All dimensions are in micrometers. 
Key features of the fabrication process are the use of thick $\mathrm{Al}$ films deposited on insulating substrates (fused silica) and patterned with a highly anisotropic dry-etching process that avoids mask undercutting thereby preserving the sharpness of corrugated electrode edges.

In a first step, $8 \mu \mathrm{m}$ of aluminum were sputter deposited from a pure Al target on 100-mm fused silica substrates. The sputtering process was realized at room temperature with a DC power of $2 \mathrm{~kW}$, leading to a deposition rate of 400 $\mathrm{nm} / \mathrm{min}$. The equipment used was a Spider-600 from Pfeiffer Vacuum. Next, a $10 \mu \mathrm{m}$-thick layer of Clariant's AZ9260 photoresist was spun and photographically defined into the mask for patterning the electrodes. Al etching was done with an inductively coupled plasma (ICP) process based on a mixed $\mathrm{Cl}_{2} / \mathrm{BCl}_{3}$ chemistry on a STS Multiplex ICP etching tool. The $\mathrm{Al}$ was etched at a rate of approximately $300 \mathrm{~nm} / \mathrm{min}$ and the selectivity toward the mask, considering a load of about $80 \%$ of the wafer surface, was observed to be close to unity. Figure $3 \mathrm{~A}$ is an optical micrograph of a fabricated device with an electrode gap of $40 \mu \mathrm{m}$, whereas Figure 3B is a SEM close-up of the patterned aluminum electrodes with smooth and vertical walls enabled by the ICP process.
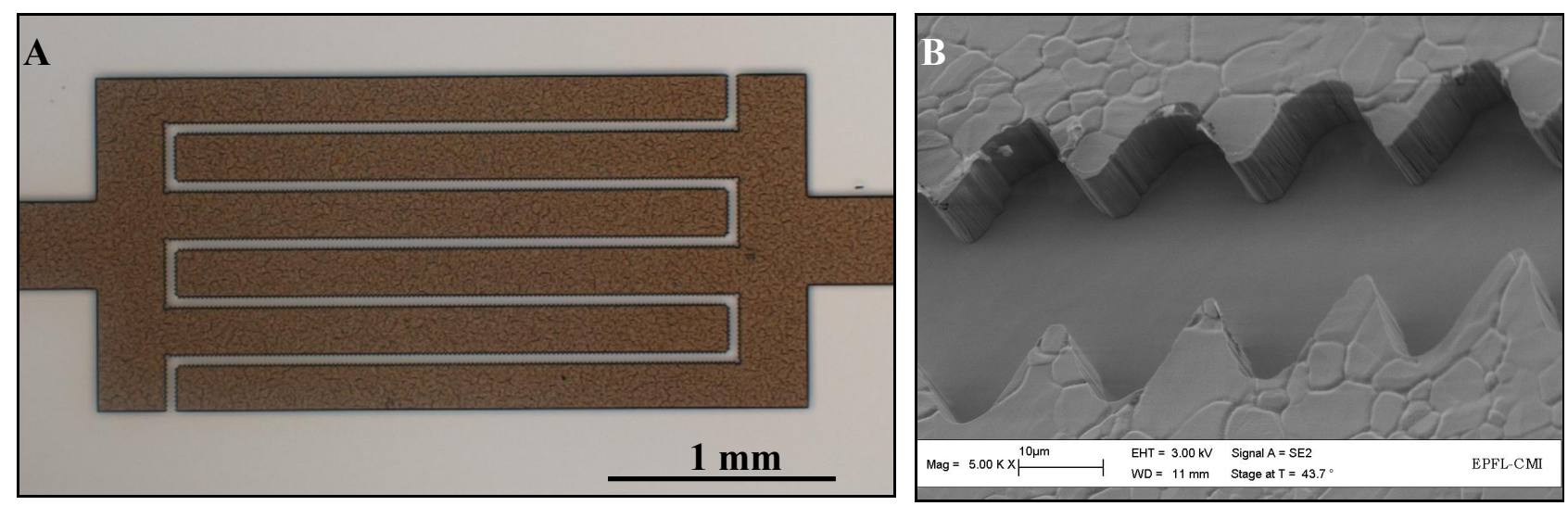

Fig. 3. A) Optical image of a fabricated device with a gap spacing of $40 \mu \mathrm{m}$. B) SEM close-up of $8 \mu \mathrm{m}$-thick dry-etched Al electrodes with corrugated edges.

\section{RESULTS AND DISCUSSION}

\subsection{Experimental setup}

The DC breakdown characterization presented in this study was performed in a small vacuum chamber (volume of $0.7 l$ ) with feedthroughs for electrical connections (MHV), pressure sensors, gas inlet, pumping and venting (see Figure 4A). Prior to each measurement session, the chamber was pumped down to a base pressure of $0.5 \mathrm{~Pa}$, then purged and filled with $\mathrm{He}, \mathrm{Ar}$ or $\mathrm{N}_{2}$. The microfabricated chips were mounted on a custom PCB with pads for wirebonds and interface to electrical contacts from the MHV feedthrough, as shown in Figure 4B and Figure 4C, respectively.

Breakdown tests were performed by measuring the current vs. voltage (I-V) characteristic of the device under test (DUT). Voltage was applied across each terminal of the DUT with a high voltage power supply (PS350 from Stanford Research Systems, Inc.) controlled by a LabView program. The voltage was increased in steps of $1 \mathrm{~V}$ every $500 \mathrm{~ms}$ from an initial value of $50 \mathrm{~V}$ until the current increased sharply and exceeded a preset threshold value. The voltage corresponding to the sharp current rise was taken as the breakdown potential, $V_{b d}[4,5]$. Depending on the pressure and electrode gap, the sharp rise in current was accompanied by a visible breakdown event, ranging from a weak glow covering the whole device footprint to a localized spark or glow. The compliance current was set at a sufficiently low threshold $(0.05 \mathrm{~mA})$ to avoid rapid destruction of the electrodes due to arcing. Paschen curves were thus plotted based on the recorded values of breakdown voltage, $V_{b d}$ vs. $p \cdot d$ for pressure ranging from $10^{2}$ to $8 \cdot 10^{4} \mathrm{~Pa}$. 

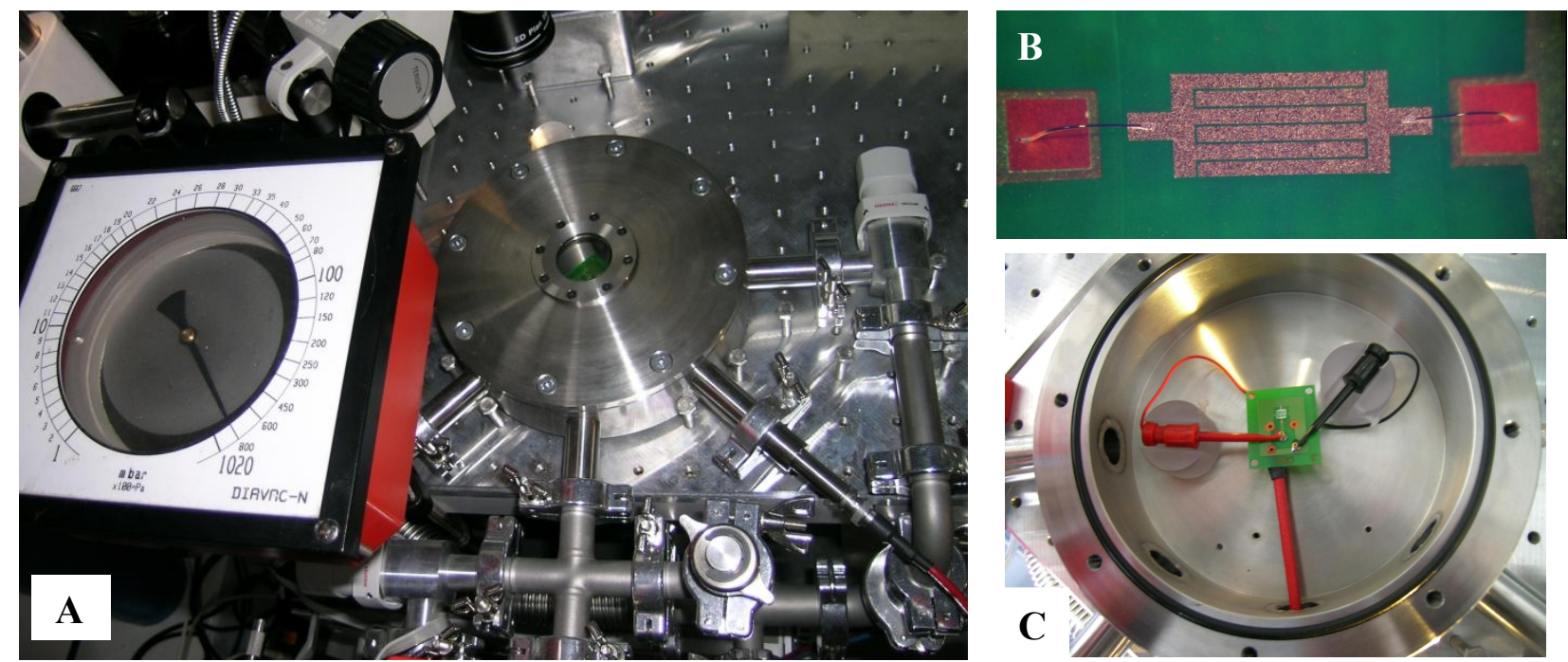

Fig. 4. A) Vacuum chamber used for the breakdown measurements, B) device under test (DUT): the microfabricated chip mounted on the PCB, and C) electrical contacts on the PCB.

\subsection{Effect of the gas type}

To introduce our Paschen curve data, it is helpful to start with an overview of the different types of behavior that were observed. Figure 5 is a plot of breakdown voltage, $V_{b d}$ vs. pressure, $p$ for three samples with an identical 100- $\mu$ m gap, in $\mathrm{N}_{2}$, Ar and He. For each pressure, the measurement was repeated five times. Solid symbols on the curves in Figure 5 represent the average of these measurements, whereas the error bars indicate lowest and highest values of the breakdown voltage.

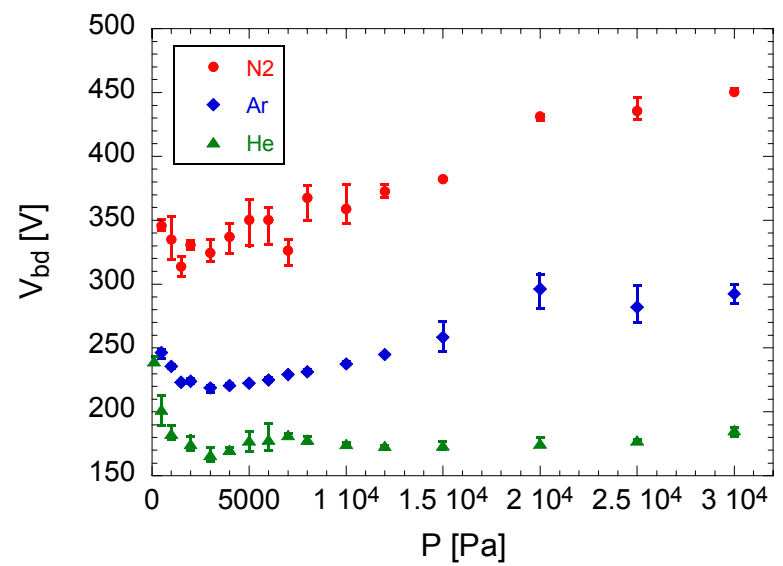

Fig. 5. Breakdown voltage, $V_{b d}$, vs. pressure, $p$ measured in different gas atmospheres $\left(\mathrm{He}, \mathrm{Ar}\right.$, and $\left.\mathrm{N}_{2}\right)$ on 3 devices with $100 \mu \mathrm{m}$ gap separation.

At first glance, typical Paschen behavior is observed, as in Figure 1, with the minimum voltage for breakdown being lower for $\mathrm{He}$, than for Ar, and for $\mathrm{N}_{2}[10,17]$. However, a more quantitative comparison of our experimental data with published values is difficult since $V_{b d}$ strongly depends on the cathode material $[9,17,18]$ and surface roughness $[5,18,19]$ and also if the breakdown is created in DC or RF mode [10,13,20]. It is reported that RF discharges exhibit significantly lower values of $V_{b d}$ than DC discharges [12]. The overall shape is as expected from the Paschen theory. However, as discussed later in section 3.6, this theory provides a poor fit when applied to the full pressure range, but provides a very good fit for higher $p \cdot d$ values. 
One important point to notice is the double dip in the He curve, which is certainly due to the different lengths scales present in the measurement setup. The dip at $3 \mathrm{kPa}$ corresponds to breakdown on the printed circuit board (gaps between wirebonds of order $3.5 \mathrm{~mm})$, while the higher pressure dip $(>10 \mathrm{kPa})$ is due to breakdown on the microfabricated electrodes. This important element will be discussed in more detail in section 3.3. The cross-over from the chip dominated regime to PCB dominated behavior basically depends on electrode spacing and on gas type: it is easily visible in $\mathrm{He}$, less noticeable in $\mathrm{Ar}$ and not observable in $\mathrm{N}_{2}$

\subsection{Effect of the gap spacing in helium}

The cross-over is clearly illustrated in Figure 6, where experimental $V_{b d}$ vs. $p$ curves are plotted in He for a sample with a $100 \mu \mathrm{m}$ gap (Figure 6A) and a $500 \mu \mathrm{m}$ gap (Figure 6B). As illustrated in Figure 1B, the pressure corresponding to the minimum $V_{b d}$ scales as $1 / d$ in the Paschen model. Both devices exhibit a minimum breakdown voltage of 160-170 V between 2 to $4 \mathrm{kPa}$ due to the the PCB, as discussed in the previous section. In addition, the device with $100 \mu \mathrm{m}$ gap has a second dip of value $175 \mathrm{~V}$ located around $15 \mathrm{kPa}$, hence for a product $p \cdot d$ of $1.5 \mathrm{~Pa} \cdot \mathrm{m}$. Published data for minimum breakdown in He reported $V_{b d}=240 \mathrm{~V}$ for $p \cdot d=5.5 \mathrm{~Pa} \cdot \mathrm{m} \mathrm{[20]} \mathrm{and} V_{b d}=150 \mathrm{~V}$ for $p \cdot d=5.33 \mathrm{~Pa} \cdot \mathrm{m}$ (or 4 torr $\cdot \mathrm{cm}$ ) [17]. In Figure 7 data of $V_{b d}$ for electrode gaps from 10 to $500 \mu \mathrm{m}$ are overlaid, as a function of pressure (Figure 7A) and $P_{r e d}=$ $p \cdot d$ (Figure 7B). For clarity, the error bars are no longer displayed. All the samples except the PCB and the chip with larger gap $(d=500 \mu \mathrm{m})$, exhibit a dip with minimum $V_{b d}$ between 170 and $210 \mathrm{~V}$. A correlation of these values of minimum $V_{b d}$ with the gap spacing cannot be found.
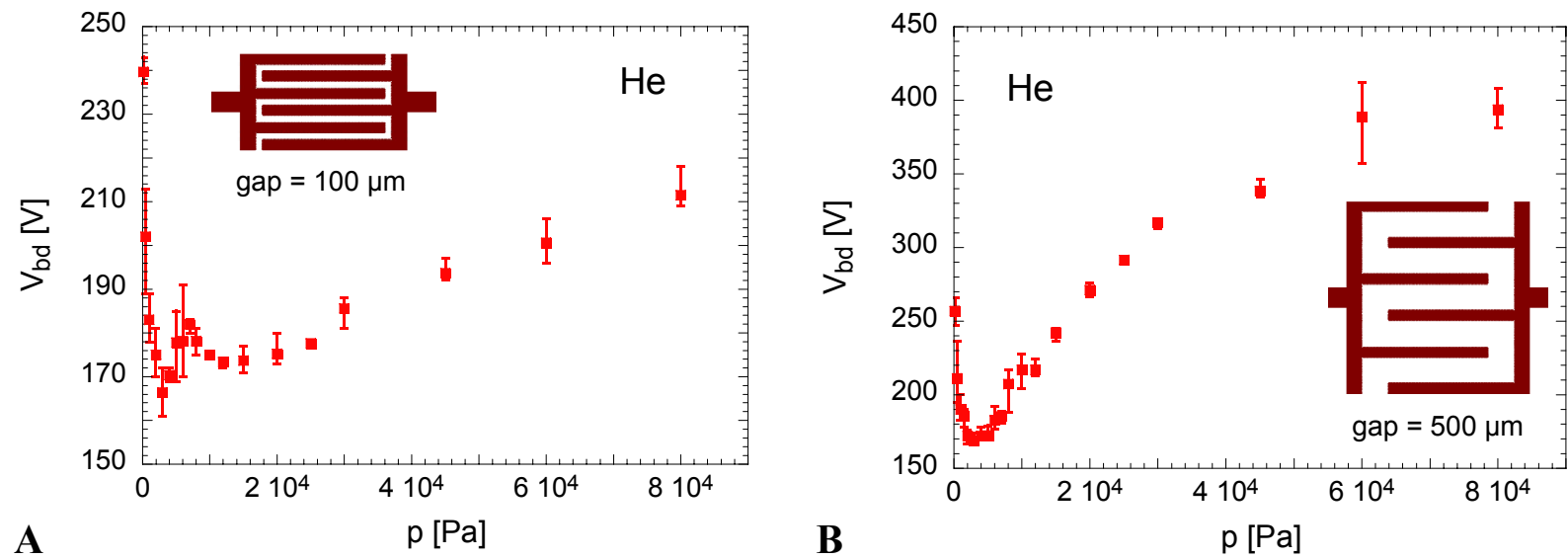

Fig. 6. Breakdown voltage, $V_{b d}$ vs. pressure, $p$ measured in helium on a device with $100 \mu \mathrm{m}$ gap separation A), and with 500 $\mu \mathrm{m}$ gap separation $\mathrm{B})$.
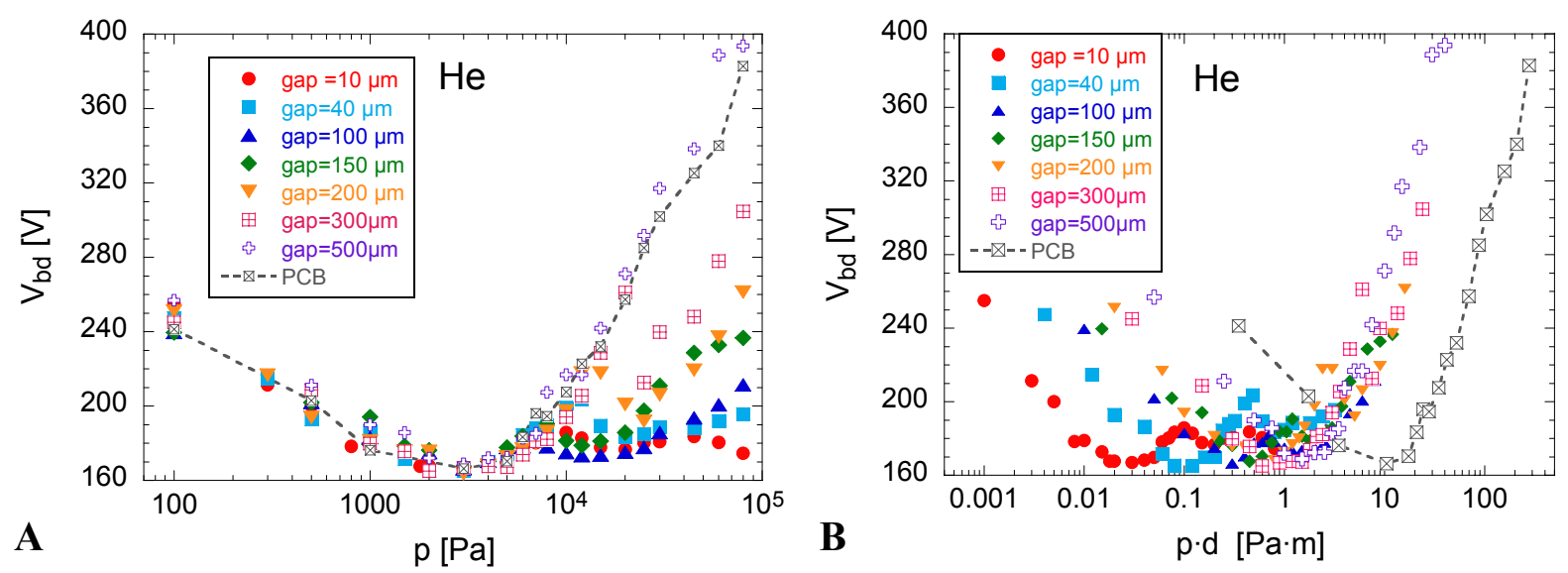

Fig. 7. A) Breakdown voltage, $V_{b d}$ vs. pressure, $p$ and B) $V_{b d}$ vs. the product $p \cdot d$ measured in He on devices with gap spacing ranging from 10 to $500 \mu \mathrm{m}$. The measured curves of the PCB are represented by a dashed line on both graphs. 


\subsection{Effect of the gap spacing in argon}

The data for breakdown in argon are presented in this section. Figure 8 is the equivalent of Figure 7 but for Ar (same gaps and pressures). Similar behavior to He is seen, but the $p \cdot d$ range where the curves overlap is larger. The cross-over from non-Paschen to Paschen behavior occurs near $1 \mathrm{~Pa} \cdot \mathrm{m}$. For small gaps the contributions from the PCB and from the chip are still clearly distinguishable. All the samples, except the PCB and the chip with larger gap $(d=500 \mu \mathrm{m})$, exhibit a dip with minimum $V_{b d}$ between 210 and $250 \mathrm{~V}$. Again, there is no correlation between these values of $V_{b d}$ minimum and the gap spacing.
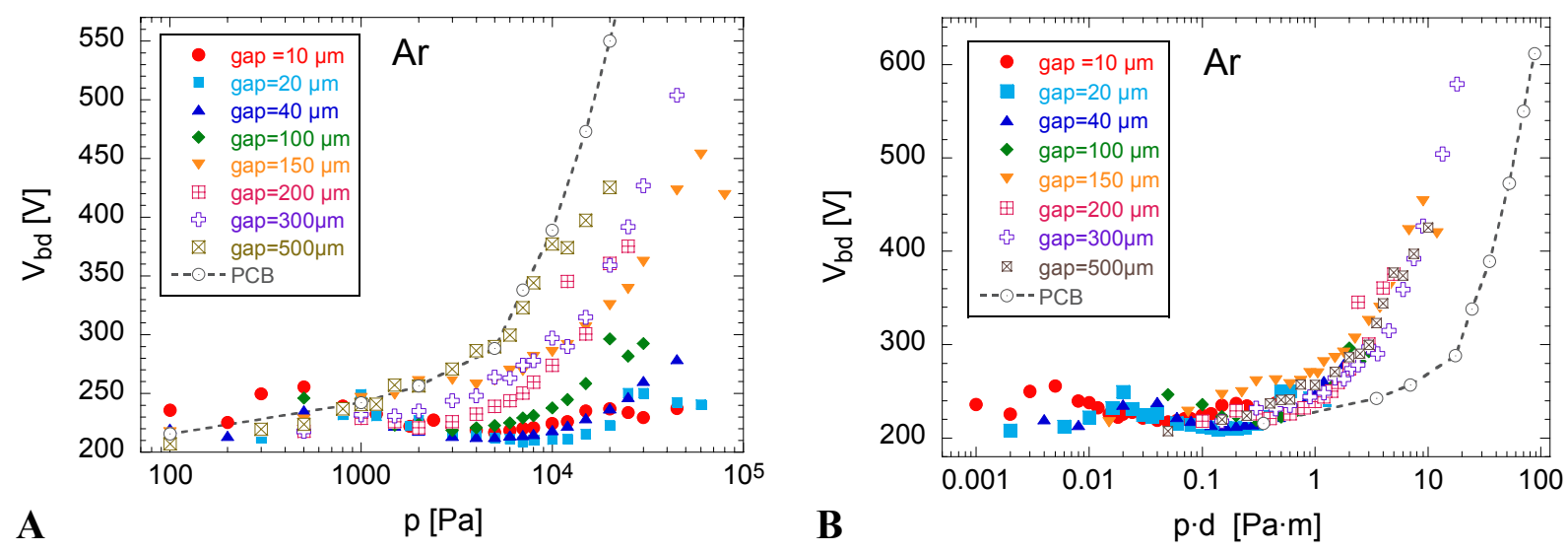

Fig. 8. A) Breakdown voltage, $V_{b d}$ vs. pressure, $p$ and B) $V_{b d}$ vs. the product $p \cdot d$ measured in Ar on devices with gap spacing ranging from 10 up to $500 \mu \mathrm{m}$. The measured curves of the PCB are represented by a dashed line.

\subsection{Effect of the gap spacing in nitrogen}

The data for breakdown in nitrogen are presented in this section. Figure 9 is the equivalent of Figure 7 and Figure 8. Less data is available for $\mathrm{N}_{2}$ than for Ar or He (fewer gaps sizes, same pressure range). However, dips in the curves are much less marked or no longer present. For nitrogen, which is one of the gases with the highest breakdown voltages, the effect of the PCB is not visible (it would occur at lower pressures than we used, i.e., below $100 \mathrm{~Pa}, 1 \mathrm{mbar}$ ). The plots fit well the overall Paschen shape, and are well represented by the reduced variable $p \cdot d$. All the measured samples exhibit minimum $V_{b d}$ between 280 and $325 \mathrm{~V}$. As expected these values are higher than for He and Ar.
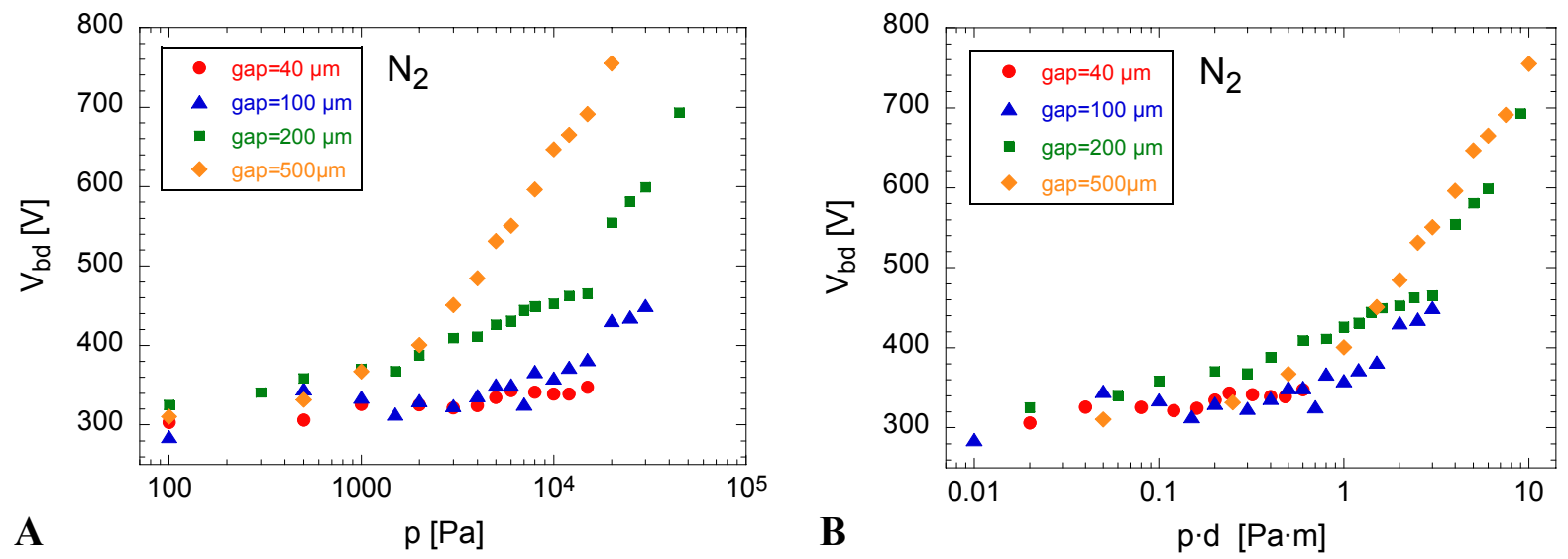

Fig. 9. A) Breakdown voltage, $V_{b d}$ vs. pressure, $p$ and B) $V_{b d}$ vs. the product $p \cdot d$ measured in $\mathrm{N}_{2}$ on devices with gap spacing ranging from 10 up to $500 \mu \mathrm{m}$. 


\subsection{Discussion}

The $V_{b d}$ vs. $p \cdot d$ plots for He, Ar and $\mathrm{N}_{2}$ in Figure 7B, Figure 8B and Figure 9B are plotted for different gaps, reflecting how the data was acquired, i.e., by varying the pressure for a fixed gap. The same data can instead be plotted grouping points taken on different samples but at a fixed pressure, emphasizing the scaling with gap rather than the scaling with pressure. This is shown in Figure 10A-C, where only the higher pressure values were kept. This mostly amounts to a zoom on the high $P_{\text {red }}$ part of Figure 7B, Figure 8B and Figure 9B.

The Paschen theory fits well experimental data in Figure 10A-C: $V_{b d}$ vs. $p \cdot d$ data points for different pressures and for gaps from 10 to $500 \mu \mathrm{m}$ fall on a single line for each gas. The solid line represents a fit to the Paschen formula (equation 1 ), restricting the fit to the higher $p \cdot d$ values. Extracting $B$ from the fit, one obtains Table 1 . The fitted $B$ is very close to the values reported in the literature for much more ideal geometry [20], which is surprising given the planar nature of our electrodes.

If instead of fitting only to the high $p \cdot d$ values one uses the literature values [20] for the Paschen parameters of Ar, one finds a very good agreement at high pressures in the linear regime (as seen above), but at low pressures the Paschen theory predicts slightly lower $V_{b d}$ than observed, by about $50 \mathrm{~V}$.

We can divide the behavior of the samples into two regimes: high $P_{\text {red }}$ where "classical" Paschen behavior is observed, and low $P_{r e d}$, where deviations are seen. A cross-over $P_{r e f}^{\text {thresh }}$ can be identified delimiting these two regimes.
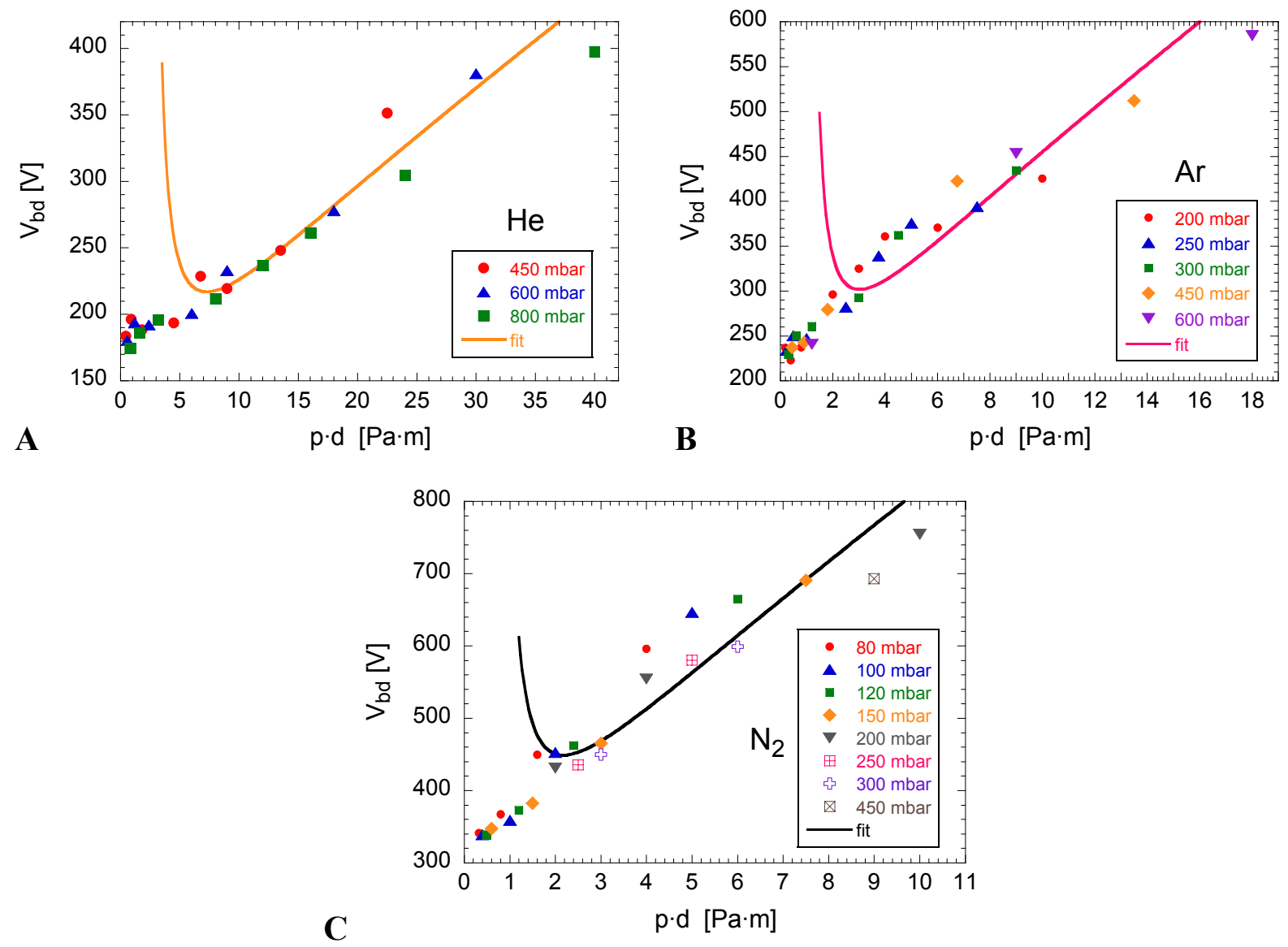

Fig. 10. Paschen curves: A) in helium, B) in argon, and C) in nitrogen. Symbols correspond to experimental data of $V_{b d}$ vs. $p \cdot d$ measured as a function of pressure on devices with different gap spacing. Lines are fits to the Paschen theory, using the points at higher $p \cdot d$ products for fitting. 
Table. 1. Values of $B$ (equation 1) from the literature [20] and from fits to experimental data in Figure 10A-C.

\begin{tabular}{|c|c|c|c|}
\hline Gas & $\begin{array}{c}\boldsymbol{B}, \text { values at DC from literature } \\
{\left[\mathrm{V} \cdot \mathrm{Pa}^{-1} \cdot \mathrm{m}^{-1}\right]}\end{array}$ & $\begin{array}{c}\boldsymbol{B}, \text { determined by fitting } \\
{\left[\mathrm{V} \cdot \mathrm{Pa}^{-1} \cdot \mathrm{m}^{-1}\right]}\end{array}$ & $\boldsymbol{\Delta B} / \boldsymbol{B}_{\boldsymbol{D C}}[\%]$ \\
\hline $\mathbf{H e}$ & 26 & 29.83 & $+14.7 \%$ \\
\hline $\mathbf{A r}$ & 135 & 100.04 & $-25.9 \%$ \\
\hline $\mathbf{N}_{\mathbf{2}}$ & 256 & 206.2 & $-19.4 \%$ \\
\hline
\end{tabular}

For helium, argon and nitrogen we have approximately $P_{r e f}^{\text {thresh }}=5,3$ and $2 \mathrm{~Pa} \cdot \mathrm{m}$, which can also be expressed as a threshold pressure. The transition occurs at lower pressures for He (Figure 7A), Ar (Figure 8A), and $\mathrm{N}_{2}$ (Figure 9A). Almost all our data for nitrogen show standard Paschen scaling.

The divergence at lower pressure has been noted for RF discharge in [12], and field emission was proposed as a mechanism. Since our gaps are larger than $10 \mu \mathrm{m}$, and since we observe the same behavior with gaps up to $500 \mu \mathrm{m}$, field emission cannot explain the observed behavior. There are two factors that might explain our data: 1) the mean free path, and 2) the planar geometry.

Figure 11 illustrates the approximate mean free path vs. pressure for our three gases. The mean free path scales as $1 / p$, and therefore one has that:

$$
\lambda \cdot p=\frac{R \cdot T}{\sqrt{2} \cdot d_{\text {atom }}^{2} \cdot N_{A v}}=\text { const }
$$

where $N_{A v}$ is Avogadro's number, $d_{\text {atom }}$ is the effective diameter of the gas atom or molecule, $T$ is the temperature and $R$ the universal gas constant.

Recall from section 1 that in order to have avalanche breakdown one must have $\lambda>>d$. The $\lambda \cdot p$ constant for He, Ar, and $\mathrm{N}_{2}$ is: $0.02,0.007$, and $0.007 \mathrm{~Pa} \cdot \mathrm{m}$. Multiplied by 500 (corresponding to requiring $\lambda>500 \cdot d$ ) one comes close to the observed $P_{\text {red }}^{\text {thresh }}$ values.

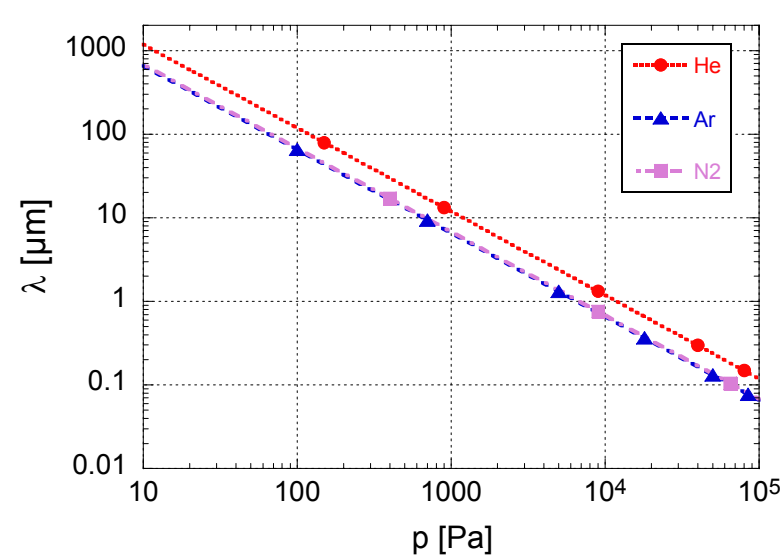

Fig. 11. Calculated main free path length $\lambda$ vs. pressure $p$ for different gases: helium, argon and nitrogen.

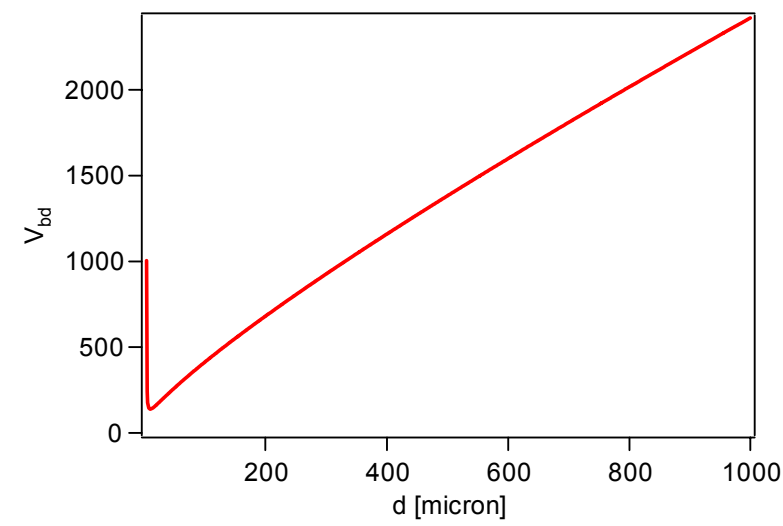

Fig. 12. Paschen curve for argon at a pressure of one atmosphere.

When measuring the breakdown between two parallel plates, there is clearly only one relevant length scale. However for a planar geometry like ours, many lengths scales are present, the smallest being the electrode gap, as shown in Figure 13. 
With a gap of $500 \mu \mathrm{m}$ and electrodes $8 \mu \mathrm{m}$ thick, we are far from parallel plates. This then allows many Paschen curves to be superposed, from the longest to the shortest length scale, as in Figure 14.

The smallest length scale is the microfabricated gap. The measured Paschen curve will then be the minimum envelope of all possible Paschen curves between the two length extremes, and will therefore have an extended flat region between the increase at low pressure for the largest scale, and the increase at high pressure for the shortest scale. This describes well the He and the Ar data, since for both those gases the effect of the PCB length scales were clearly visible. For nitrogen, one would have to work below $500 \mathrm{~Pa}$ (5 mbar) to see any effect of the PCB, so we end up with nearly standard Paschen curves in $\mathrm{N}_{2}$.

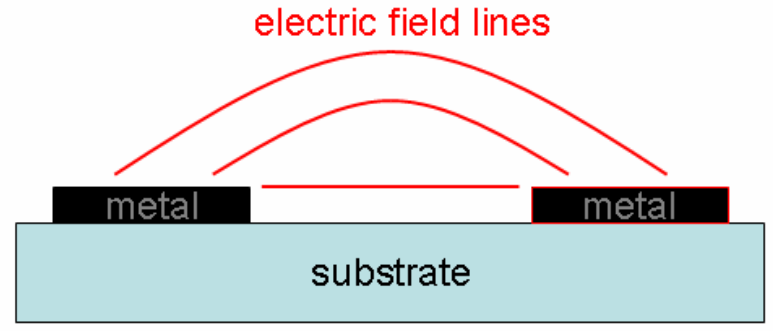

Fig. 13. Illustration of three different length scales for the electric field in a planar geometry.

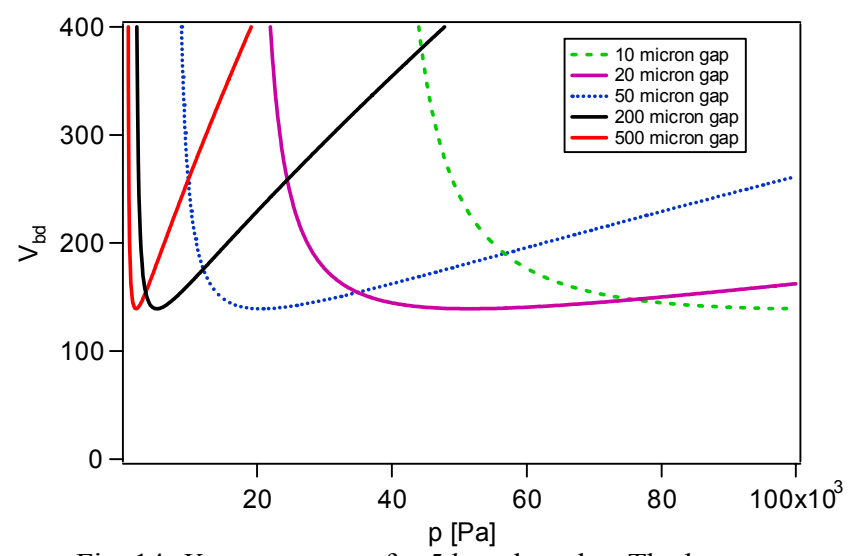

Fig. 14. $V_{b d}$ vs. pressure for 5 length scales. The larger the gaps, the smaller the pressure where $V_{b d}$ is minimum. If all the length scales are present, the measured Paschen curve will be the minimum envelope of all the curves.

When operating at one atmosphere, the situation is somewhat simpler with respect to the different length scales. The Paschen curve data taken at one atmosphere in [4] do no show this multiple length scales. Figure 12 is a plot of $V_{b d}$ vs. gap size in argon (same parameters as in Figure 1, but plotted for a fixed pressure of $10^{5} \mathrm{~Pa}$ ). At this high pressure, the minimum of the Paschen curve is pushed down to approximately $5 \mu \mathrm{m}$. In this case it is not surprising that for gaps larger than $10 \mu \mathrm{m}$, it is the smallest gap that dictates the breakdown voltage. The fact that the geometry is planar is then of no consequence.

\section{CONCLUSION}

An experimental study was presented of the breakdown voltage of microfabricated 8- $\mu \mathrm{m}$ thick aluminum electrodes with gaps ranging from 10 to $500 \mu \mathrm{m}$. The pressure was varied from 500 to $8.10^{4} \mathrm{~Pa}$ (5 to $800 \mathrm{mbar}$ ) in helium, argon and nitrogen atmospheres. The breakdown voltage was plotted as a function of the Paschen reduced variable $P_{\text {red }}=p \cdot d$. For higher values of pressure or gap (high $P_{\text {red }}$ ), classical Paschen scaling is observed for all three gases. For lower values of $P_{r e d}$ however, significant deviations are seen, particularly at low pressures. These differences cannot be attributed to field emission, since the effect occurs at gaps of several hundred microns.

At low values of $P_{\text {red }}$ the mean free path becomes of order the gap spacing, which explains the slightly higher than expected breakdown voltages seen in this range. The many length scales effectively present in our planar geometry (onchip and even off-chip), that lead to the superposition of several Paschen curves, explain the difficulty in using the Paschen theory to fit over the full $p \cdot d$ range. Taking the many length scales into account allows explaining the three regimes in the $V_{b d}$ vs. pressure curves for a fixed electrode gap: at low pressures the curve is given by the Paschen theory computed using the largest spacing in the system, at intermediate pressures $V_{b d}$ is constant and corresponds to the minimum in the Paschen curve for that gas and electrode material, and at higher pressures the curve is the standard Paschen curve using the smallest gap in the system. 
In order to ignite a DC plasma on-chip, the $V_{b d}$ must be exceeded. This voltage is gas dependent, and significantly lower in He than in $\mathrm{N}_{2}$. The lowest $V_{b d}$ values in He are obtained for electrodes with serrated patterns and range from 170 to $210 \mathrm{~V}$. While a smaller electrode gap does increase the maximum electric field, at low pressures this is not particularly relevant in view of the Paschen theory and especially in view of the many length scales present in the planar geometry. For igniting plasmas at low pressures, comparable ignition voltages are found for a wide range of gap sizes, and the electrode spacing can then be driven by other considerations. If breakdown is to be avoided, one must note that lower pressures lead to lower breakdown voltages, and that therefore the minimum safe operation voltage for a given gap is decreased over a large pressure range linearly from the value at one atmosphere.

At pressures below one atmosphere, care must therefore be taken when applying the Paschen formula to gaps in the micron range. For operation at one atmosphere standard Paschen behavior is seen for gaps larger than 10 microns.

\section{REFERENCES}

1 R. S. Dhariwal, J. M. Torres, and M. P. Y. Desmulliez, "Electric field breakdown at micrometre separations in air and nitrogen at atmospheric pressure", IEE Proc. Sci. Meas. Technol. 147 (5), pp. 261-265, 2000.

2 J.-M. Torres and R. S. Dhariwal, "Electric field breakdown at micrometre separations", Nanotechnology 10, pp. 102-107, 1999.

3 P. G. Slade and E. D. Taylor, "Electrical breakdown in atmospheric air between closely spaced $(0.2 \mu \mathrm{m}-40 \mu \mathrm{m})$ electrical contacts", IEEE Trans. Comp. Packaging Technol. 25 (3), pp. 390-396, 2002.

4 A. Wallash and L. Levit, "Electrical breakdown and ESD phenomena for devices with nanometer-to-micron gaps", Proc. of SPIE 4980, pp. 87-96, 2003.

5 C.-H. Chen, J. A. Yeh, and P.-J. Wang, "Electrical breakdown phenomena for devices with micron separations", $J$. Micromech. Microeng. 16, pp. 1366-1373, 2006.

6 F. W. Strong, J. L. Skinner, P. M. Dentinger, and N. C. Tien, "Electrical breakdown across micron scale gaps in MEMS structures", Proc. of SPIE 6111, pp. 611103, 2006.

7 F. Paschen, "Über die zum Funkenübergang in Luft, Wasserstoff und Kohlensäure bei verschiedenen Drucken erforderliche Potentialdifferenz", Annalen der Physik 273 (5), pp. 69-96, 1889.

8 J. Townsend, Electricity in Gases, Oxford University Press, New York, 1915.

$9 \quad$ H. Jacobs and A. P. LaRocque, "Minimum sparking potentials of barium, magnesium, and aluminum in argon", $J$. Appl. Phys. 18, pp. 199-203, 1947.

10 L. Baars-Hibbe, P. Sichler, C. Schrader, N. Lucas, K.-H. Gericke and S. Büttgenbach, "High frequency glow discharges at atmospheric pressure with micro-structured electrode arrays", J. Phys. D: Appl. Phys. 38, pp. 510-517, 2005.

11 N. St. J. Braithwaite, "Introduction to gas discharges", Plasma Sources Sci. Technol. 9, pp. 517-527, 2000.

12 L. Baars-Hibbe, P. Sichler, C. Schrader, C. Gessner, K.-H. Gericke, and S. Büttgenbach, "Micro-structured electrode arrays: atmospheric pressure plasma processes and applications", Surf. Coat. Technol. 174-175, pp. 519-523, 2003.

13 C. Schrader, L. Baars-Hibbe, K.-H. Gericke, E. M. van Veldhuizen, N. Lucas, P. Sichler, and S. Büttgenbach, "Micro-structured electrode arrays: glow discharges in $\mathrm{Ar}$ and $\mathrm{N}_{2}$ at atmospheric pressure using a variable radio frequency generator", Vacuum 80, pp. 1144-1148, 2006.

14 T. Ono, D. Y. Sim, and M. Esashi, "Micro-discharge and electric breakdown in a micro-gap", J. Micromech. Microeng. 10, pp. 445-451, 2000.

$15 \mathrm{H}$. Schlemm and D. Roth, "Atmospheric plasma processing with microstructure electrodes and microplanar reactors", Surf. Coat. Technol. 142-144, pp. 272-276, 2001.

16 P. Carazzetti, Ph. Renaud, and H. R. Shea, "Low-power hermetically sealed on-chip plasma light sources micromachined in glass," Tech. Dig. IEEE Int. Conf. MEMS'08, January 13-18, Tucson, AZ, pp. 818-821, 2008.

17 M. A. Hassouba, F. F. Elakshar, and A. A. Garamoon, "Measurement of the breakdown potentials for different cathode materials in the Townsend regime", Fizika A 11 (2), pp. 81-90, 2002.

18 H. Jacobs and A. P. LaRocque, "The role of the cathode surface in sparking phenomena", Physical Review 74 (2), pp. 163-165, 1948.

19 L. H. Germer, "Electrical breakdown between close electrodes in air", J. Appl. Phys. 30 (1), pp. 46-51, 1959.

20 Y. P. Raizer, Gas Discharge Physics, Springer, Berlin, Germany, 1997. 